\title{
Imaging of Atherosclerosis in Apoliprotein E Knockout Mice: Targeting of a Folate-Conjugated Radiopharmaceutical to Activated Macrophages
}

\author{
Wilfredo Ayala-López, Wei Xia, Bindu Varghese, and Philip S. Low \\ Department of Chemistry, Purdue University, West Lafayette, Indiana
}

\begin{abstract}
Early detection of heart disease is essential for the implementation of intervention strategies that reduce the risk of cardiovascular events. Radioimaging methods that have been explored for this purpose include ${ }^{18} \mathrm{~F}-\mathrm{FDG}$, which measures sites of elevated metabolic activity; ${ }^{99 m T c-a n n e x i n ~ A 5, ~ w h i c h ~ r e v e a l s ~ r e g i o n s ~ o f ~}$ enhanced apoptosis and thrombosis; and 99mTc-labeled antilectinlike oxidized low-density lipoprotein receptor 1 antibody, which detects the lectinlike oxidized low-density lipoprotein receptor 1 that is overexpressed on a variety of vasculatureassociated cells. In this study, we examine the use of a folatetargeted chelate of ${ }^{99 m T c}$, termed ${ }^{99 m T c-E C 20}$, for imaging of
\end{abstract} folate receptor (FR)-expressing macrophages that accumulate in atherosclerotic plaques, internalize cholesterol-rich lipoprotein particles, and evolve into foam cells that form components of vulnerable atherosclerotic lesions. Methods: $99 \mathrm{mTC}-\mathrm{EC} 20$ was injected into apoliprotein $E$ knockout (apoE-/-) mice fed a normal or Western (high-fat) diet for 25 wk and imaged by $\gamma$-scintigraphy. Treated mice were also dissected, and radioactivities in excised aortas were quantified by $\gamma$-counting and imaged by autoradiography. The role of FR-expressing macrophages in uptake of $99 \mathrm{mTC}-\mathrm{EC} 20$ was also examined by comparing images of apoE-/- mice before and after treatment with clodronate liposomes to deplete tissue macrophages, comparing the sites of $99 \mathrm{mTC}-\mathrm{EC} 20$ enrichment with sites of macrophage accumulation in thin sections of atherosclerotic tissues, and examining the expression of FRs on atherosclerotic plaquederived macrophages by flow cytometry. Results: ApoE-/- mice on Western chow exhibited significantly greater accumulation of ${ }^{99 m}$ Tc-EC20 in atherosclerotic lesions than their counterparts on normal chow. The aortas of apoE-/- mice on a Western diet demonstrated greater numbers of FR-positive macrophages by flow cytometry than did those of apoE-/- mice on a normal diet. Clodronate liposome treatment significantly reduced the accumulation of $99 \mathrm{mTC}-\mathrm{EC} 20$ in atherosclerotic tissues, suggesting that macrophages or monocytes are responsible for uptake of the folate-linked radioimaging agent. Histologic and autoradiographic analysis of tissue sections demonstrated that macrophage accumulation correlated with regions of ${ }^{99 m}$ Tc-EC20

Received Oct. 4, 2009; revision accepted Jan. 26, 2010.

For correspondence or reprints contact: Philip S. Low, Department of Chemistry, Purdue University, 560 Oval Dr., West Lafayette, IN 47907. E-mail: plow@purdue.edu

COPYRIGHT $\odot 2010$ by the Society of Nuclear Medicine, Inc. uptake. Conclusion: ${ }^{99 m}$ Tc-EC20 can be used for the imaging of atherosclerosis by selectively targeting FR-positive activated macrophages.

Key Words: atherosclerosis-associated macrophages; imaging of vulnerable plaque; folate receptor targeting; EC20

J Nucl Med 2010; 51:768-774

DOI: 10.2967/jnumed.109.071324

Card ardiovascular events associated with progressive atherosclerosis constitute the main cause of death in Western societies today (1). Atherosclerosis originates from chronic inflammation of the arteries characterized by enhanced infiltration of leukocytes, uptake of lipoprotein particles, proliferation of smooth muscle and endothelial cells, and apoptosis of foam cells (2). Myocardial infarction, the most serious of the atherosclerotic sequelae, can result from expansion and destabilization of atherosclerotic lesions, leading to plaque rupture and subsequent thrombotic events (2). Prevention of serious cardiovascular events has, therefore, not surprisingly been linked to early diagnosis and detection of vulnerable atherosclerotic lesions (3).

Vascular properties that are thought to differ between vulnerable and quiescent atherosclerotic lesions generally include the inflammatory status, morphology, degree of stenosis, cap thickness and stability, and proteinase activity of the atherosclerotic plaque (3). Attempts to quantitate these differences as a means of assessing cardiovascular risk have focused on the design of noninvasive imaging agents that detect the accumulation of immune cells (4), expression of metalloproteinase activity (5), heightened consumption of glucose $(6)$, and apoptosis of cells within the vascular bed (7). Although such strategies show considerable promise for identification of vulnerable plaque, they also suffer from some degree of nonspecificity because each strategy also images healthy cells involved in other processes. 
In an effort to add another tool to the arsenal of methods for the detection of vulnerable plaque, we have undertaken the imaging of sites of accumulation of activated macrophages within the vasculature. Activated macrophages are thought to constitute key players in the development of vulnerable plaque $(8,9)$, because they accumulate within the intima at sites of vascular damage; take up large quantities of lipoprotein particles, eventually becoming macrophage foam cells; discharge inflammatory cytokines and growth factors that promote the influx and proliferation of other cells; and release metalloproteinases and reactive oxygen species that can cause plaque weakening and rupture (2). For this reason, the macrophage has emerged as an important diagnostic and therapeutic target for atherosclerosis $(9,10)$.

A major distinction between activated and quiescent macrophages lies in their expression of a folate receptor (FR) $(11,12)$. Thus, FR $\beta$, a glycosylphosphatidylinositolanchored glycoprotein, is expressed on the surface of activated macrophages (11-13) but essentially absent from the surfaces of resting macrophages and other immune cells. As a consequence, FR-targeted imaging agents have been used to image sites of inflammation in a variety of inflammatory diseases in both animals and humans. ${ }^{99 \mathrm{~m} T c-}$ EC20, a folate-targeted radiopharmaceutical, has been particularly useful for imaging sites of macrophage accumulation in both rheumatoid arthritis and osteoarthritis $(12,14,15)$. In this study, however, we evaluate for the first time-to our knowledge-whether ${ }^{99 \mathrm{~m} T c-E C 20}$ can be used as an effective imaging agent for atherosclerosis. Using apolipoprotein E knockout (apoE-/-) mice raised on both normal and high-fat diets, we documented selective uptake of ${ }^{99 \mathrm{~m} T c-E C 20}$ by macrophages present within atherosclerotic lesions, and we demonstrated the ability to detect atherosclerosis in live mice by whole-animal radioimaging.

\section{MATERIALS AND METHODS}

\section{Animals}

All animal procedures were approved by the Purdue Animal Care and Use Committee in accordance with guidelines from the National Institutes of Health. ApoE-/- breeding trios (Jackson Laboratories) were maintained in a temperature- and humiditycontrolled room on a 12-h dark-light cycle. Female offspring were weaned at $3 \mathrm{wk}$ of age and either maintained on normal rodent chow or transferred at $5 \mathrm{wk}$ of age to a Western diet consisting of $2 \%$ cholesterol and $21.2 \%$ fat (Harlan-Teklad), as indicated in the figure legends.

\section{Preparation of ${ }^{99 m}$ Tc-EC20}

EC20 vials were a kind gift from Endocyte, Inc. ${ }^{99 m}$ Tc-EC20 was prepared as described elsewhere (16). Briefly, vials containing lyophilized EC20 were heated at $100^{\circ} \mathrm{C}$ for $5 \mathrm{~min}$, after which $2 \mathrm{~mL}$ of a $925 \mathrm{MBq} / \mathrm{mL}$ solution of sodium pertechnetate (Cardinal Health) was added, and the vial was heated for an additional $15 \mathrm{~min}$. After dilution with the desired volume of saline, mice were injected intraperitoneally with either $400 \mu \mathrm{L}$ of imaging agent (18.5 MBq, $250 \mathrm{nmol}$ of EC20 per kilogram) or the same volume of imaging agent supplemented with a 100 -fold molar excess of free folic acid (to compete for unoccupied FRs). Unbound ${ }^{99 \mathrm{~m}} \mathrm{Tc}-\mathrm{EC} 20$ was allowed to clear from the tissues for $4 \mathrm{~h}$ before imaging.

Imaging and Assessment of ${ }^{99 m}$ Tc-EC20 Accumulation in ApoE-/- Mice

ApoE-/- mice on a normal or Western diet were anesthetized with 3\% isoflurane and imaged using a Kodak Image Station operated with Kodak molecular imaging software (version 4.5; Carestream Molecular Imaging). Abdomens were shielded with a 5-mm-thick lead shield to mask radioactivities emanating from the kidneys and the bladder. Both radiographic images and radioimages had a focus setting of $7 \mathrm{~mm}$ and a field of view of $200 \times 200 \mathrm{~mm}$. Radioimages were acquired for $1 \mathrm{~min}$ using a radioisotopic phosphor screen (Carestream Molecular Imaging), no illumination source, a $4 \times 4$ binning setting, and an f-stop of 0 . Radiographic images were acquired for $55 \mathrm{~s}$ using a Kodak radiographic phosphor screen (Carestream Molecular Imaging) and used to coregister anatomic structures with radioisotopic signals during overlays. The following settings were used for radiographs: energy of $35 \mathrm{kVp}$, current of $149 \mu \mathrm{A}$, no x-ray filter, no illumination source, and f-stop of 4 . The signal was quantitated using region-of-interest analysis. Net intensities were recorded and plotted using GraphPad Prism software (version 4; GraphPad Software).

For quantitation of the accumulation of ${ }^{99 \mathrm{~m}} \mathrm{Tc}-\mathrm{EC} 20$ in mouse aortas and heart tissues, mice were euthanized and thoracic aortas excised. Radioactivities were counted for 2 min using a $\gamma$-counter (Packard). Results are reported as percentage injected dose per gram of tissue.

\section{Autoradiography and Histology}

To image areas of accumulation of ${ }^{99 \mathrm{~m}} \mathrm{Tc}-\mathrm{EC} 20$ in atherosclerotic aortas, apoE $-/-$ mice on a normal or Western diet were injected with ${ }^{99 \mathrm{~m} T c-E C 20}$ and euthanized, and thoracic aortas were excised. For cross-sections, aortic arches were embedded in optimal-cutting-temperature compound and frozen in liquid nitrogen. Serial sections were cut with a Leica CM1800 cryostat and placed on polylysine-coated microscope slides (Thermo Scientific). Either whole aortas or aortic arch cross-sections $(40 \mu \mathrm{m})$ were exposed to a phosphor screen for $18 \mathrm{~h}$ at $4^{\circ} \mathrm{C}$. The phosphor screen was read using a Typhoon phosphor imager (GE Healthcare) at a resolution of $50 \mu \mathrm{m}$.

Aortic tissue sections (10 $\mu \mathrm{m}$ thick) directly adjacent to those used for autoradiography were used for histology. Hematoxylin and eosin staining was performed to visualize lesion morphology. Staining with a macrophage-specific monoclonal antibody (Mac3/CD107b; eBioscience Inc.) was conducted as follows. Aortic arch sections were fixed with zinc-buffered formalin for $10 \mathrm{~min}$, and endogenous biotin and peroxidase activity were blocked. Sections were incubated with antimouse CD107b antibody (1:50 dilution) for $1 \mathrm{~h}$; after washing, sections were then incubated with goat antirat biotinylated antibody (KPL Protein Research Products) at a 1:500 dilution for $30 \mathrm{~min}$. After the second washing, streptavidin-horseradish peroxidase (BD Pharmingen) was added for an additional $30 \mathrm{~min}$. Slides were developed with diaminobenzidine (BD Pharmingen) according to the manufacturer's instructions. Negative controls consisted of slides developed in the absence of primary antibody. An Olympus BH-2 microscope 
coupled with a charge-coupled device camera was used to obtain all photomicrographs.

\section{Treatment of Mice with Clodronate Liposomes}

Phosphate-buffered saline (PBS) and clodronate liposomes were synthesized as described (17). Briefly, $86 \mathrm{mg}$ of egg phosphatidylcholine plus $8 \mathrm{mg}$ of cholesterol were dissolved in 1:1 chloroform:methanol. Solvent was evaporated using a rotoevaporator for $15 \mathrm{~min}$, and the resulting film was rehydrated with PBS or a $0.6 \mathrm{M}$ solution of clodronate (Sigma) in PBS for $2 \mathrm{~h}$. Resulting multilamellar vesicles were sonicated for $3 \mathrm{~min}$ and allowed to swell for $2 \mathrm{~h}$ at $25^{\circ} \mathrm{C}$. Liposomes were washed 3 times with PBS by centrifugation at $100,000 \mathrm{~g}$ for $30 \mathrm{~min}$ and resuspended in $4 \mathrm{~mL}$ of PBS. Liposomes were extruded 5 times through both a 400- and 200-nm-pore-size polycarbonate filter and stored at $4{ }^{\circ} \mathrm{C}$ until use. The resulting liposomes consisted of a $7: 1.3$ molar ratio of egg phosphatidylcholine:cholesterol. The efficiency of clodronate entrapment using this method is $7.8 \%$ (17).

For systemic elimination of macrophages, apoE-/- mice were fed a Western diet for $8 \mathrm{wk}$, after which $200 \mu \mathrm{L}$ of PBS or clodronate liposomes ( $4 \mathrm{mg}$ of clodronate per dose) were injected intraperitoneally daily for $5 \mathrm{~d}$. After treatment, mice were injected intraperitoneally with ${ }^{99 \mathrm{~m}} \mathrm{Tc}-\mathrm{EC} 20$ and imaged, as described above.

\section{Digestion of Aortas and Flow Cytometry}

ApoE-/- mice on a normal or Western diet for $25 \mathrm{wk}$ were euthanized, and their thoracic aortas were dissected. Aortas were transferred to folate-deficient RPMI 1640 (Invitrogen) containing $12.5 \%$ fetal bovine serum, $1 \%$ phosphatidylserine, $1 \mathrm{mg}$ of collagenase type II (Sigma) per milliliter, and $1 \mathrm{mg}$ of elastase type IV (Sigma) per milliliter (18). Aortas were incubated for $2 \mathrm{~h}$ at $37^{\circ} \mathrm{C}$, with gentle swirling of the suspension every $30 \mathrm{~min}$. Cells were washed 3 times with fresh folate-deficient RPMI 1640 and resuspended in the same medium in preparation for flow cytometric analyses.

The resulting cell suspensions were incubated for $1 \mathrm{~h}$ at $37^{\circ} \mathrm{C}$ in a 1:50 dilution of polyclonal rabbit anti-FR antibody (FL-257; Santa Cruz Biotechnologies). After washing, a 1:100 dilution of fluorescein isothiocyanate-conjugated antirabbit antibody (Sigma) and a 1:100 dilution of tricolor anti-F4/80 monoclonal antibody (eBioscience) were added and incubated for an additional hour at $37^{\circ} \mathrm{C}$. Cells were washed, resuspended in PBS, and analyzed in a FACSCalibur flow cytometer (BD Bioscience). Cells were analyzed using CellQuant software (version 3.5; BD Biosciences).

\section{Statistical Analysis}

Statistical significance among experimental groups was calculated using $t$ tests. Values of $P$ less than 0.05 were considered significant.

\section{RESULTS}

99mTc-EC20 Targets Atherosclerotic Aortas of ApoE-/Mice by Binding to FR

The development of atherosclerosis in apoE-/- mice can be significantly accelerated by maintaining the mice on a high-fat (Western) diet $(19,20)$. To evaluate the ability of 99mTc-EC20 to image atherosclerotic lesions, apoE-/mice were fed either normal or Western chow for $25 \mathrm{wk}$, injected intraperitoneally with ${ }^{99 \mathrm{~m}} \mathrm{Tc}-\mathrm{EC} 20$, and then analyzed by radioimaging. As seen in Figures $1 \mathrm{~A}$ and $1 \mathrm{~B}$, apoE $-/-$ mice fed a Western diet, compared with apoE-/- mice maintained on normal rodent chow, exhibited an average increase of approximately $70 \%$ in ${ }^{99 m}$ Tc-EC20 signal intensity in the aortocardiac region. Importantly, when similar atherosclerotic mice on a Western diet were preinjected with a 100-fold excess of free folic acid to compete with ${ }^{99 \mathrm{~m}} \mathrm{Tc}-\mathrm{EC} 20$ for binding to FRs, the signal intensity was reduced to near-background levels (Fig. 1B). These data suggest that atherosclerotic lesions are enriched in FR-positive cells and that uptake of ${ }^{99 m}$ Tc-EC20 is FR-mediated.

Previous studies have demonstrated that the major sites of atherosclerotic lesion development in apoE $-/-$ mice occur in the aortic root, aortic arch, and associated branching arteries (19). To assess whether ${ }^{99 \mathrm{~m}}$ Tc-EC20 was in fact
FIGURE 1. 99mTc-EC20 targets aortas of apoE-/- mice and can be used as imaging agent for atherosclerosis. ApoE-/- mice were fed either normal or Western diet for $25 \mathrm{wk}$ and then injected intraperitoneally with either $99 \mathrm{~m}$ Tc-EC20 or ${ }^{99 m}$ Tc-EC20 plus $100-$ fold excess free folic acid. Radioimages were obtained on Kodak Imaging Station (A) $(n=10)$, and regions of interest were quantitatively analyzed using instrument software (B) $(n=10)$. Mice were then euthanized, and excised aortas were analyzed for radioactivity by $\gamma$-counting (C; $n=5)$. During imaging, 5-mm lead shields were used to cover abdomens to avoid interference from ${ }^{99 m T c-E C 20}$ uptake in kidneys and bladder. Data in $\mathrm{C}$ are presented as mean $\pm \mathrm{SD}$. ${ }^{*} P<0.05$. $\mathrm{FA}=$ folic acid.

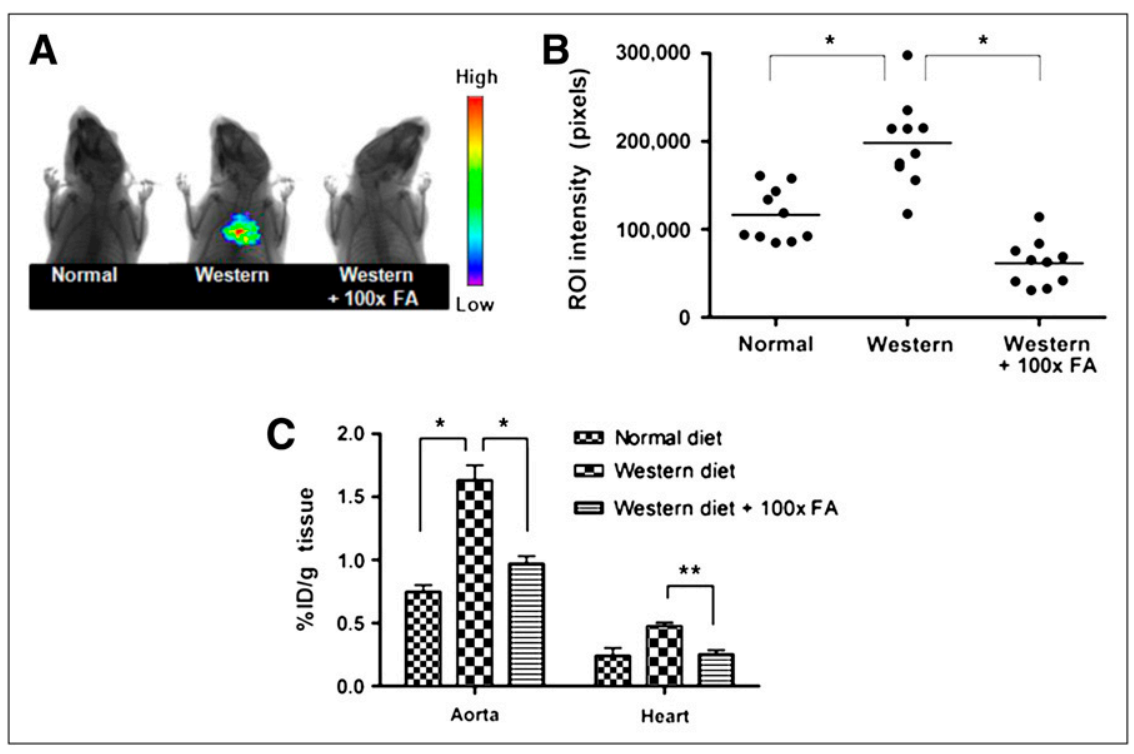


targeting these regions of enhanced atherosclerosis, thoracic aortas and hearts were dissected, and accumulation of ${ }^{99 \mathrm{~m} T c-E C 20}$ in the resected tissues was quantitated by $\gamma$-counting. As shown in Figure 1C, ${ }^{99 \mathrm{~m} T c-E C 20}$ uptake was 3-fold lower in the hearts than in the aortas, and accumulation in the aortas was approximately $120 \%$ higher in mice on Western chow than on a normal diet. Moreover, competition with excess folic acid, compared with noncompeted controls, decreased ${ }^{99 \mathrm{~m}} \mathrm{Tc}-\mathrm{EC} 20$ retention in the aortas by $41 \%$ (Fig. 1C).

To further assess the specificity of ${ }^{99 \mathrm{~m}} \mathrm{Tc}-\mathrm{EC} 20$ in targeting atherosclerotic aortas, uptake of ${ }^{99 \mathrm{~m} T c-E C 20}$ in the aortas of a different set of similarly treated apoE-1mice was examined by autoradiography. As shown in Figure 2, aortas of mice on a Western diet showed significantly greater uptake in the aortic root and arch than those of mice fed a normal diet. However, aortas from apoE-/- mice on normal chow also exhibited uptake in their aortic roots, albeit at a lower level — that is, consistent with the observation that apoE-/- mice spontaneously develop atherosclerotic lesions even on a normal diet $(19,20)$. Also, as seen previously, when mice fed the Western diet were administered a 100 -fold greater dose of free folic acid than ${ }^{99 \mathrm{~m} T c-E C 20}$, the radioactivity in the aortic root and arch was significantly reduced (Fig. 2), suggesting again that uptake is FR-mediated.

Activated FR-Positive Macrophages Are Responsible for Uptake of $99 \mathrm{~m} T \mathrm{c}-\mathrm{EC} 20$ in Atherosclerotic Plaque

Because accumulation of activated macrophages constitutes a central step in the pathogenesis of atherosclerosis $(2,21)$, and because activated macrophages express high levels of FR (11-13), we explored whether systemic depletion of macrophages might reduce uptake of ${ }^{99 \mathrm{~m}} \mathrm{Tc}-$ EC20 in atherosclerotic lesions. For this purpose, apoE-/mice maintained on a Western diet were treated with

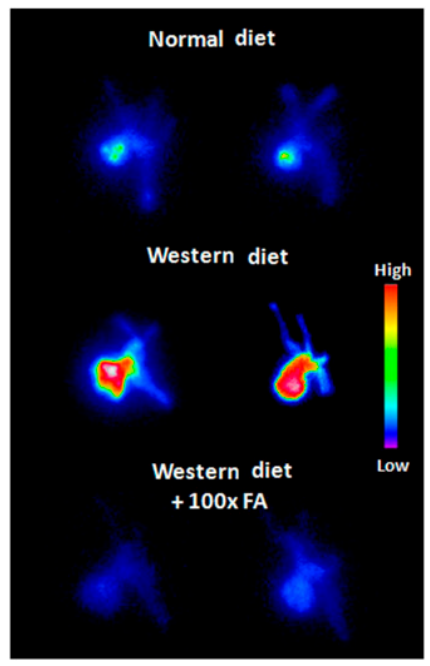

FIGURE 2. 99mTc-EC20 targets aortic root and arch of apoE-/- mice. ApoE-/- mice fed normal or Western diet for $25 \mathrm{wk}$ were injected with $99 \mathrm{mTc}$ EC20, and thoracic aortas were excised after allowing $4 \mathrm{~h}$ for clearance of radiopharmaceutical from FRnegative tissues. Aortas were exposed to phosphor screen and images developed using a phosphor imager. Images of aortas of mice on normal diet, aortas of mice on Western diet, and aortas of mice on Western diet but preinjected with 100-fold dose of free folic acid (FA) before injection of $99 \mathrm{mTC}-\mathrm{EC} 20$. clodronate liposomes to systemically eliminate macrophages (22). Then, mice were injected with ${ }^{99 \mathrm{~m} T c-E C 20}$ and imaged. As shown in Figure 3, clodronate liposome treatment reduced uptake of ${ }^{99 \mathrm{~m} T c-E C 20}$ by $65 \%$ relative to mice injected with analogous PBS-containing liposomes. These data suggest that macrophages are primarily responsible for uptake of ${ }^{99 \mathrm{~m} T c-E C 20}$ in the atherosclerotic lesions. The data are also consistent with the previously demonstrated role of FR-positive macrophages in mediating uptake of ${ }^{99 \mathrm{~m}} \mathrm{Tc}-\mathrm{EC} 20$ in rheumatoid arthritic and osteoarthritic joints $(12,14,15,23)$.

To further establish that macrophages are responsible for accumulation of ${ }^{99 \mathrm{~m}} \mathrm{Tc}-\mathrm{EC} 20$ in atherosclerotic lesions, we injected apoE-/- mice on a Western diet with ${ }^{99 \mathrm{~m} T c-}$ EC20 and examined serial sections of their aortas by autoradiography and histochemistry. Consecutive sections were stained with hematoxylin and eosin to reveal vascular morphology, labeled with Mac-3/CD107b to localize sites

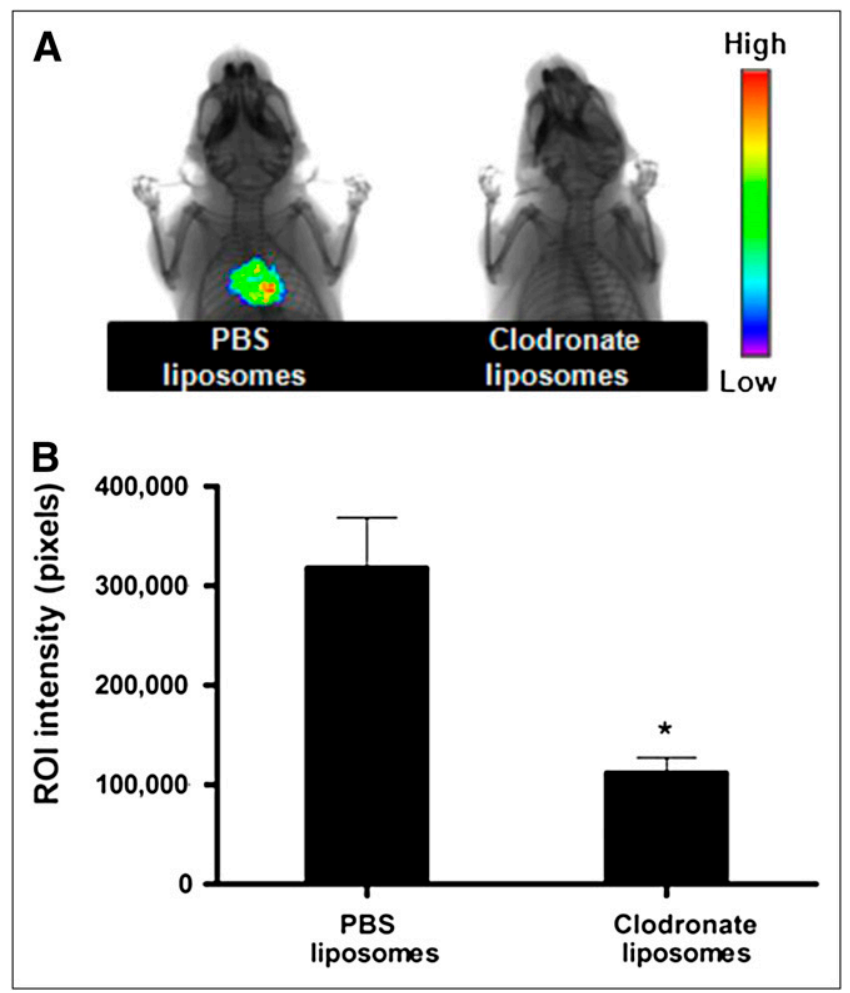

FIGURE 3. Treatment of apoE-/- mice on Western diet with clodronate liposomes diminishes uptake of $99 \mathrm{mTC}$ EC20. ApoE-/- mice on Western diet for 8 wk were treated for $5 \mathrm{~d}$ with single injections of PBS or clodronate liposomes (4 mg of clodronate/injection) intraperitoneally. One day later, ${ }^{99 \mathrm{~m} T c-E C 20}$ was injected intraperitoneally, and animals were imaged $4 \mathrm{~h}$ later to assess cardiovascular uptake of radiopharmaceutical (A). Regions of interest (ROIs) were then quantitatively analyzed using instrument software (B) $(n=5)$. In all cases, 5-mm lead shields were used to cover abdomen to avoid interference from ${ }^{99 \mathrm{mT}}$ T-EC20 uptake in kidneys and bladder. Data are presented as mean \pm SD. ${ }^{\star} P<0.05$. 
of macrophage enrichment, and imaged by autoradiography to identify locations of ${ }^{99 m}$ Tc-EC20 accumulation. As seen in Figure 4 and Supplemental Figure 1 (supplemental materials are available online only at http://jnm.snmjournals. org), areas of high macrophage content and atherosclerotic lesion formation invariably corresponded with loci of elevated ${ }^{99 \mathrm{~m}}$ Tc-EC20 uptake.

\section{Macrophages Isolated from Aortas of ApoE-/- Mice Express FR}

To confirm by yet another method that FR-positive macrophages play the major role in mediating accumulation of ${ }^{99 \mathrm{~m}} \mathrm{Tc}-\mathrm{EC} 20$ in the aortas of apoE-/- mice, thoracic aortas were digested to obtain single-cell suspensions, and cells expressing a macrophage marker (F4/80) were analyzed by flow cytometry for simultaneous expression of FR. As seen in Supplemental Figure 2, F4/80positive macrophages were found to comprise $1.1 \%$ and $3.0 \%$ of all cells in the thoracic aortas of mice fed a normal diet and Western diet, respectively. This diet-dependent increase in macrophage content was not unexpected, because an increase in monocyte or macrophage infiltration has been established to constitute a hallmark of atherogenesis $(2,10,21)$. More important, macrophages from mice fed a normal diet were only $11 \%$ FR-positive, whereas macro-
A Mac-3

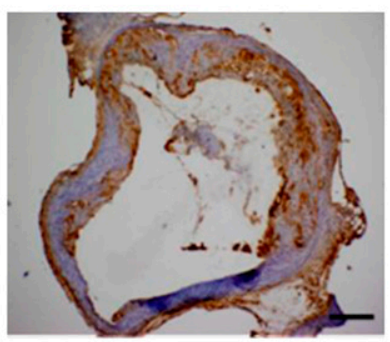

B

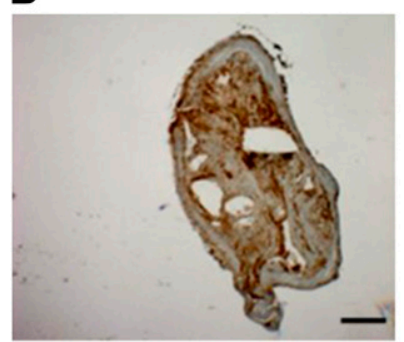

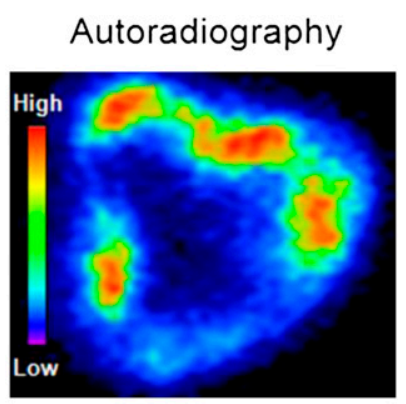

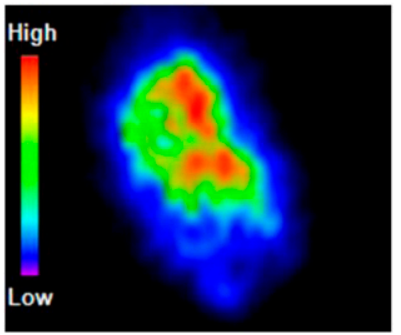

FIGURE 4. 99mTc-EC20 preferentially accumulates in areas of high macrophage content within atherosclerotic plaques of apoE-/- mice. ApoE-/- mice on Western diet for 25 wk were injected with $99 \mathrm{mT}$ T-EC20. After 4-h tissue clearance period, aortas were dissected and embedded in optimal-cutting-temperature medium. Sections of ascending aorta and brachiocephalic artery were prepared and exposed to phosphor screen for $18 \mathrm{~h}$. Images were taken using phosphor imager. Consecutive sections were used for Mac-3 immunohistochemistry and hematoxylin and eosin staining (Supplemental Fig. 1). Bar $=100 \mu \mathrm{m}$. phages from mice on the Western diet were 33\% FRpositive, suggesting that the high-fat diet not only increased total macrophage content but also tripled the percentage of FR-positive macrophages (Fig. 5). Given that FR expres-

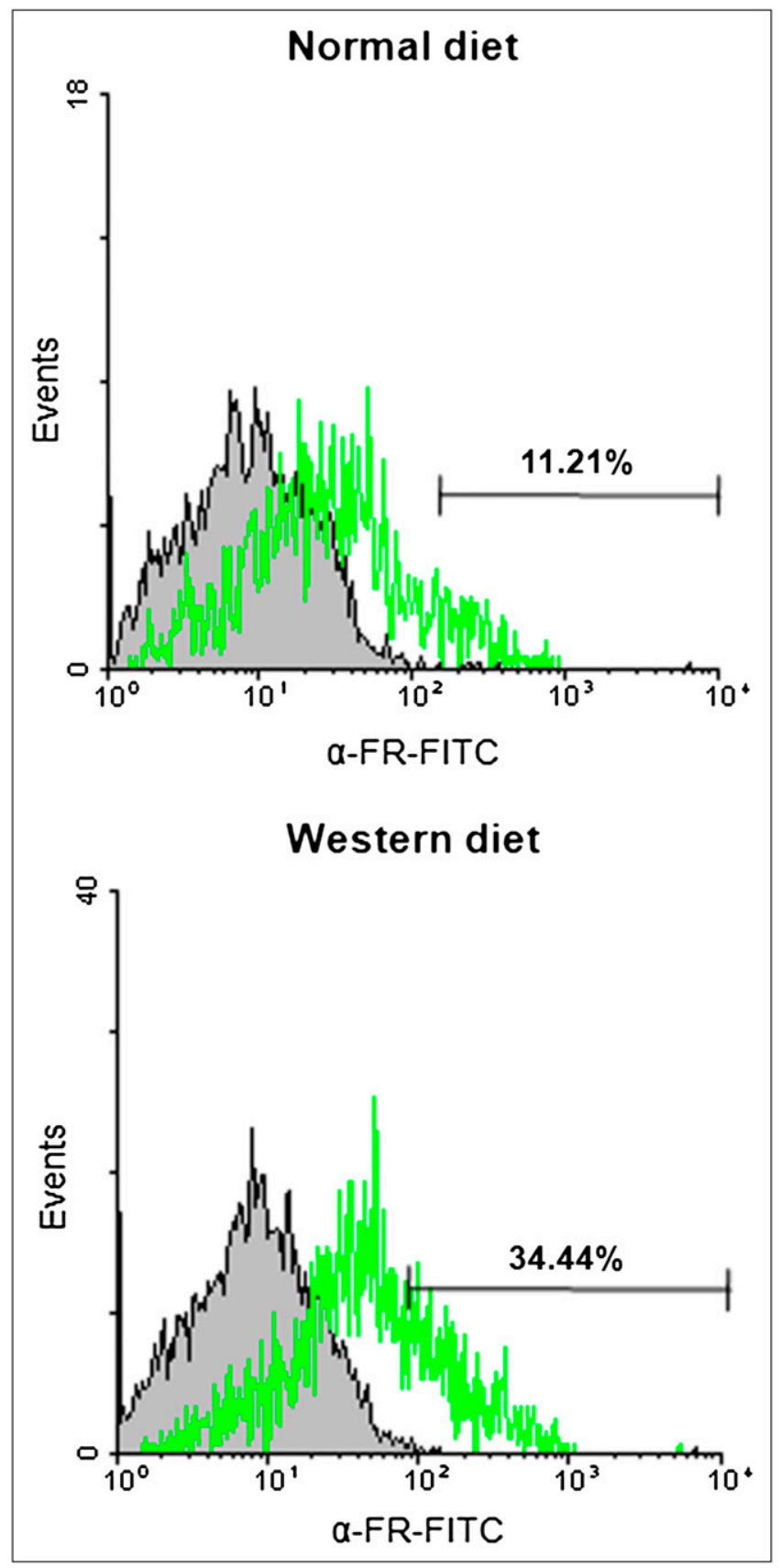

FIGURE 5. Percentage increase in FR-positive macrophage numbers in apoE-/- mice on Western diet. ApoE-/- mice were fed normal or Western diet for 25 wk. Mice were euthanized and thoracic aortas excised and digested with collagenase and elastase. Resulting cell suspensions were analyzed by flow cytometry after incubation with tricolorconjugated F4/80 antibody (macrophage marker) and rabbit anti-FR primary antibody followed by fluorescein isothiocyanate-conjugated (FITC) antirabbit IgG secondary antibody. 
sion constitutes a marker for macrophage activation $(11,12)$, these data suggest that the higher-fat diet elevates both the number and the activation state of plaque macrophages.

\section{DISCUSSION}

In this study, we have shown that folate-targeted 99mTc-EC20 can be used to image atherosclerotic lesions in apoE- $/-$ mice. Uptake of ${ }^{99 \mathrm{~m} T c-E C 20}$ was demonstrated to be concentrated primarily in the aortic arch, where the largest accumulation of atherosclerotic plaque is known to occur (19). Accretion of the radionuclide was also found to be FR-mediated, as accumulation at sites of plaque deposition was inhibited by preadministration of excess free folic acid. Finally, uptake of ${ }^{99 \mathrm{~m}}$ Tc-EC20 was demonstrated to be diet-dependent, because mice on a Western diet displayed significantly more accumulation of ${ }^{99 m}$ Tc-EC20 than mice on a normal diet.

Overexpression of accessible FR has been previously documented to occur in cancer cells and activated macrophages $(11,12,15,24,25)$. Accumulation of ${ }^{99 m}$ Tc-EC20 in murine atherosclerotic lesions was shown to be macrophage-mediated. Thus, regions of ${ }^{99 \mathrm{~m} T c-E C 20}$ enrichment were found to correlate with sites of macrophage concentration (Fig. 4), accumulation of ${ }^{99 \mathrm{~m}}$ Tc-EC20 in atherosclerotic lesions was shown to be suppressed by elimination of macrophages with clodronate liposomes, and analyses of the macrophages derived from atherosclerotic regions demonstrated that many stained positively with FR-specific antibodies (Fig. 5). These observations are consistent with previous histologic data on atherosclerotic heart valves from Syrian golden hamsters, which suggested that lipidladen macrophage foam cells can be readily targeted with folate-Texas Red (26).

It has been proposed recently that several types of macrophages likely reside concurrently within atherosclerotic lesions. For instance, it has been reported that human carotid lesions may contain both M1 and M2 macrophages $(27,28)$ and that the more stable regions of the plaque are enriched in macrophages that exhibit antiinflammatory properties, whereas the more labile loci may be characterized by accumulation of inflammatory macrophages (29). Because FR-positive macrophages have been demonstrated to be proinflammatory $(12,23,30)$, it will be informative to explore whether the abundance of FR-positive macrophages at sites of cardiovascular disease might correlate with plaque vulnerability. If such a correlation were to emerge, it would provide the motivation to explore various folatetargeted therapeutics for the treatment of unstable lesions that might give rise to serious cardiovascular events. In this respect, the total macrophage content of atherosclerotic plaques has been associated repeatedly with plaque vulnerability $(8,9,31,32)$.

Noninvasive methods for the assessment of plaque vulnerability have traditionally been preferred over intra- vascular approaches, and although noninvasive methodologies currently under development for the evaluation of atherosclerosis show considerable promise, ${ }^{99 \mathrm{~m} T c-E C 20}$ may offer advantages not shared by these alternative strategies. Thus, although ${ }^{18} \mathrm{~F}-\mathrm{FDG}$ has been used for most atherosclerosis imaging applications to date (33), ${ }^{18} \mathrm{~F}-\mathrm{FDG}$ accumulates in any metabolizing cell, leading to possible regions of high image intensity at sites unrelated to atherosclerosis. ${ }^{99 \mathrm{~m}} \mathrm{Tc}-\mathrm{EC} 20$ accumulation, in contrast, is limited to sites enriched in activated macrophages $(14,16)$, significantly reducing the potential for image misinterpretation. Radiolabeled antibodies directed to specific antigens (e.g., lectinlike oxidized low-density lipoprotein receptor 1 and benzodiazepine receptor) within an atherosclerotic lesion have also been used for plaque imaging $(4,34)$. However, concerns over target specificity, rate of clearance from off-target tissues, efficiency of plaque penetration, and image intensity may limit use of monoclonal antibodies in these imaging applications $(4,6,35,36)$. Finally, lipid-binding proteins such as annexin A5 $(7,37)$ that recognize exposed phosphatidylserine on apoptotic cells can also be exploited to image dying cells, including apoptotic macrophage foam cells in atherosclerotic lesions. However, because phosphatidylserine can be exposed on any dying or damaged cell, including activated platelets; ischemic, stressed, or traumatized cardiomyocytes; myoblasts; immune cells; and erythrocytes $(38,39)$, opportunities for misinterpretation of data once again arise. Although 99mTc-EC20 will admittedly image virtually any site of active inflammation, its ease of synthesis and radiolabeling, excellent tissue penetration, and rapid clearance from FR-negative tissues (due to its small size and water solubility $(16,25)$, lack of toxicity or immunogenicity $(12,24)$, and specificity for an abundant cell type in atherosclerotic lesions [macrophages] $(12,14,23))$ renders it a possible candidate for further evaluation.

Finally, together with the previously published data on osteoarthritis (12) and rheumatoid arthritis (14,23), the evidence presented here documents a third inflammatory disease that can be visualized with an FR-targeted radioimaging agent $(26,40)$. Because many other inflammatory diseases are also characterized by a localized accumulation of activated macrophages, it will be important to determine whether similar folate conjugates might prove useful in imaging these other inflammatory diseases as well.

\section{CONCLUSION}

This study demonstrates that FR-positive macrophages accumulate in atherosclerotic lesions of apoE $-/-$ mice and that the folate-derivatized radiopharmaceutical ${ }^{99 m}$ Tc-EC20 can selectively target loci of activated macrophages present in atherosclerotic lesions. The ability of $99 \mathrm{~m}$ Tc-EC20 to target FR-positive macrophages in atherosclerotic plaques suggests that this radiopharmaceutical may be useful for noninvasive imaging of vulnerable atherosclerotic lesions. 


\section{ACKNOWLEDGMENT}

This work was supported by a research grant from Endocyte, Inc.

\section{REFERENCES}

1. Ross R. Atherosclerosis: an inflammatory disease. N Engl J Med. 1999;340:115_ 126.

2. Libby P. Inflammation in atherosclerosis. Nature. 2002;420:868-874.

3. Langer HF, Haubner R, Pichler BJ, Gawaz M. Radionuclide imaging: a molecular key to the atherosclerotic plaque. J Am Coll Cardiol. 2008;52:1-12.

4. Fujimura Y, Hwang PM, Trout Iii H, et al. Increased peripheral benzodiazepine receptors in arterial plaque of patients with atherosclerosis: an autoradiographic study with $\left[{ }^{3} \mathrm{H}\right] \mathrm{PK}$ 11195. Atherosclerosis. 2008;201:108-111.

5. Hartung D, Schafers M, Fujimoto S, et al. Targeting of matrix metalloproteinase activation for noninvasive detection of vulnerable atherosclerotic lesions. Eur J Nucl Med Mol Imaging. 2007;34(suppl 1):S1-8.

6. Laurberg JM, Olsen AK, Hansen SB, et al. Imaging of vulnerable atherosclerotic plaques with FDG-microPET: no FDG accumulation. Atherosclerosis. 2007;192: 275-282.

7. Zhao Y, Kuge Y, Zhao S, et al. Comparison of ${ }^{99 \mathrm{~m}} \mathrm{Tc}$-annexin A5 with ${ }^{18} \mathrm{~F}-\mathrm{FDG}$ for the detection of atherosclerosis in apoE-1- mice. Eur J Nucl Med Mol Imaging. 2007;34:1747-1755.

8. Halvorsen B, Otterdal K, Dahl TB, et al. Atherosclerotic plaque stability: what determines the fate of a plaque? Prog Cardiovasc Dis. 2008;51:183-194.

9. Tiwari RL, Singh V, Barthwal MK. Macrophages: an elusive yet emerging therapeutic target of atherosclerosis. Med Res Rev. 2008;28:483-544.

10. Choudhury RP, Lee JM, Greaves DR. Mechanisms of disease: macrophagederived foam cells emerging as therapeutic targets in atherosclerosis. Nat Clin Pract Cardiovasc Med. 2005;2:309-315.

11. Nakashima-Matsushita N, Homma T, Yu S, et al. Selective expression of folate receptor beta and its possible role in methotrexate transport in synovial macrophages from patients with rheumatoid arthritis. Arthritis Rheum. 1999;42: 1609-1616.

12. Xia W, Hilgenbrink AR, Matteson EL, Lockwood MB, Cheng JX, Low PS. A functional folate receptor is induced during macrophage activation and can be used to target drugs to activated macrophages. Blood. 2009;113:438-446.

13. van der Heijden JW, Oerlemans R, Dijkmans BA, et al. Folate receptor $\beta$ as a potential delivery route for novel folate antagonists to macrophages in the synovial tissue of rheumatoid arthritis patients. Arthritis Rheum. 2009;60:12-21.

14. Turk MJ, Breur GJ, Widmer WR, et al. Folate-targeted imaging of activated macrophages in rats with adjuvant-induced arthritis. Arthritis Rheum. 2002;46: 1947-1955.

15. Matteson EL, Lowe VJ, Prendergast FG, et al. Assessment of disease activity in rheumatoid arthritis using a novel folate targeted radiopharmaceutical Folatescan. Clin Exp Rheumatol. 2009;27:253-259.

16. Leamon CP, Parker MA, Vlahov IR, et al. Synthesis and biological evaluation of EC20: a new folate-derived, ${ }^{99 \mathrm{~m}} \mathrm{Tc}$-based radiopharmaceutical. Bioconjug Chem. 2002;13:1200-1210.

17. Love WG, Camilleri JP, Williams BD. Efficient clodronate entrapment within multilamellar and unilamellar liposomes. J Pharmacol Toxicol Methods. 1992; 27:185-189.

18. Ray JL, Leach R, Herbert JM, Benson M. Isolation of vascular smooth muscle cells from a single murine aorta. Methods Cell Sci. 2001;23:185-188.

19. Nakashima Y, Plump AS, Raines EW, Breslow JL, Ross R. ApoE-deficient mice develop lesions of all phases of atherosclerosis throughout the arterial tree. Arterioscler Thromb. 1994;14:133-140.
20. Jawien J, Nastalek P, Korbut R. Mouse models of experimental atherosclerosis. J Physiol Pharmacol. 2004;55:503-517.

21. Shashkin P, Dragulev B, Ley K. Macrophage differentiation to foam cells. Curr Pharm Des. 2005;11:3061-3072.

22. Buiting AM, Zhou F, Bakker JA, van Rooijen N, Huang L. Biodistribution of clodronate and liposomes used in the liposome mediated macrophage 'suicide' approach. J Immunol Methods. 1996;192:55-62.

23. Paulos CM, Varghese B, Widmer WR, Breur GJ, Vlashi E, Low PS. Folatetargeted immunotherapy effectively treats established adjuvant and collageninduced arthritis. Arthritis Res Ther. 2006;8:R77.

24. Low PS, Henne WA, Doorneweerd DD. Discovery and development of folicacid-based receptor targeting for imaging and therapy of cancer and inflammatory diseases. Acc Chem Res. 2008;41:120-129.

25. Reddy JA, Xu LC, Parker N, Vetzel M, Leamon CP. Preclinical evaluation of ${ }^{99 \mathrm{~m} T c-E C 20}$ for imaging folate receptor-positive tumors. J Nucl Med. 2004;45: 857-866.

26. Antohe F, Radulescu L, Puchianu E, Kennedy MD, Low PS, Simionescu M. Increased uptake of folate conjugates by activated macrophages in experimental hyperlipemia. Cell Tissue Res. 2005;320:277-285.

27. Bolick DT, Skaflen MD, Johnson LE, et al. G2A Deficiency in mice promotes macrophage activation and atherosclerosis. Circ Res. 2009;104:318-327.

28. Charo IF. Macrophage polarization and insulin resistance: PPAR $\gamma$ in control. Cell Metab. 2007;6:96-98.

29. Bouhlel MA, Derudas B, Rigamonti E, et al. PPAR $\gamma$ activation primes human monocytes into alternative M2 macrophages with anti-inflammatory properties. Cell Metab. 2007;6:137-143

30. Varghese B, Haase N, Low PS. Depletion of folate-receptor-positive macrophages leads to alleviation of symptoms and prolonged survival in two murine models of systemic lupus erythematosus. Mol Pharm. 2007;4:679-685.

31. San Miguel Hernandez A, Inglada-Galiana L, Garcia Iglesias R, Alonso Castillejos N, Martin Gil FJ. Soluble CD40 ligand: a potential marker of cardiovascular risk [in Spanish]. Rev Clin Esp. 2007;207:418-421.

32. Broz P, Ben-Haim N, Grzelakowski M, Marsch S, Meier W, Hunziker P. Inhibition of macrophage phagocytotic activity by a receptor-targeted polymer vesicle-based drug delivery formulation of pravastatin. J Cardiovasc Pharmacol. 2008;51:246-252.

33. Nahrendorf M, Zhang H, Hembrador S, et al. Nanoparticle PET-CT imaging of macrophages in inflammatory atherosclerosis. Circulation. 2008;117:379-387.

34. Ishino S, Mukai T, Kuge Y, et al. Targeting of lectinlike oxidized low-density lipoprotein receptor 1 (LOX-1) with ${ }^{99 \mathrm{~m} T c-l a b e l e d}$ anti-LOX-1 antibody: potential agent for imaging of vulnerable plaque. J Nucl Med. 2008;49:16771685 .

35. de Wolf FA, Brett GM. Ligand-binding proteins: their potential for application in systems for controlled delivery and uptake of ligands. Pharmacol Rev. 2000;52: 207-236.

36. Laitinen I, Marjamaki P, Haaparanta M, et al. Non-specific binding of $\left[{ }^{18} \mathrm{~F}\right] \mathrm{FDG}$ to calcifications in atherosclerotic plaques: experimental study of mouse and human arteries. Eur J Nucl Med Mol Imaging. 2006;33:1461-1467.

37. Laufer EM, Reutelingsperger CP, Narula J, Hofstra L. Annexin A5: an imaging biomarker of cardiovascular risk. Basic Res Cardiol. 2008;103:95-104.

38. van Genderen HO, Kenis H, Hofstra L, Narula J, Reutelingsperger CP. Extracellular annexin A5: functions of phosphatidylserine-binding and twodimensional crystallization. Biochim Biophys Acta. 2008;1783:953-963.

39. Boersma HH, Kietselaer BL, Stolk LM, et al. Past, present, and future of annexin A5: from protein discovery to clinical applications. J Nucl Med. 2005;46:20352050.

40. Chen WT, Mahmood U, Weissleder R, Tung CH. Arthritis imaging using a nearinfrared fluorescence folate-targeted probe. Arthritis Res Ther. 2005;7:R310R317. 